\section{Topical administration of Esculetin as a potential therapy for experimental dry eye syndrome}

D Jiang ${ }^{1}, X \mathrm{Liu}^{1}$ and $\mathrm{J} \mathrm{Hu}$ pathway and enhanced the therapeutic effect of CsA.

Eye (2017) 31, 1724-1732; doi:10.1038/eye.2017.117; published online 23 June 2017

\section{Introduction}

Dry eye syndrome (DES) is a multifactorial disease of tears and ocular surface that results in symptoms of discomfort, visual disturbance and tear film instability. It is accompanied by increased osmolarity of the tear film and inflammation of the ocular surface. ${ }^{1}$ There are many etiological factors of DES including chronic diseases, infectious, aging, radiation and environmental factors like smoking or video display. ${ }^{2}$ According to the Dry Eye Workshop (DEWS) report in 2007, prevalence of dry eye ranges from 5 to $30 \%$ in people aged 50 years and older. ${ }^{1}$ Cyclosporine A (CsA) is one kind of drug proved therapeutic by FDA in curing DES through inhibiting the immune reaction and inflammation of the ocular surface. However, topical CsA has potential side effects, for example, ocular burning, conjunctival hyperemia, epiphora, eye pain, foreign body sensation, ${ }^{3}$ especially when used chronically. Appropriately administration of this agent and monitoring for side effects mentioned above remained tough problems. In addition, topical CsA eyedrops were not easily available in our country and many patients cannot afford the high price of it. These illuminated us to seek for lower cost, more effective or less side effect drugs for DES.

Esculetin, which was extracted from Chinese herb drug 'Qinpi', was known for inducing apoptosis in human cancer cells, ${ }^{4}$ attenuated the expression of pro-inflammatory mediators in the inflamed colon ${ }^{5}$ and inhibiting VEGF-induced cell proliferation and DNA synthesis with no cell toxicity both in vitro and in vivo ${ }^{6}$ in recent studies. Researches on Esculetin were systemic
Received: 11 August 2016 Accepted in revised form: 27 April 2017 Published online: 23 June 2017
Department of

Hosptial, Shanghai

Chinese Medicine,

Correspondence: Ophthalmology, Longhua University of Traditional South Wan Shanghai 200032, Chin Fax: +862164875390. E-mail:drliuxinquan@

${ }^{1} T$ These authors contributed equally to this work.

\begin{abstract}
Both topical Esculetin and Esculetin combing $(77 \pm 12 / \mathrm{HP}, 92 \pm 12 / \mathrm{HP}, P<0.05)$, decreased FL scores $(7.48 \pm 0.33,5.09 \pm 0.24, P<0.05)$ at week similar trend. In Western blot analysis, downregulations of $\mathrm{p}$-ERK1/2 were observed in therapy groups when compared with group DES control and the most decreasing was found in group $C \& E(P<0.05)$.

Conclusion Topical Esculetin improved DES symptoms, downregulated the inflammatory cytokine expressions, suppressed the ERK1/2
\end{abstract}


administration in mice or rats, while topical Esculetin in curing ocular diseases had not ever been reported before. In this study, we aimed to investigate whether topical Esculetin can improve the DES symptoms and prevent the damage on ocular surface via cytokine expressions and signal pathways. We also intended to observe whether Esculetin is capable of enhance the therapeutic effects of CsA, which may give a chance of reducing the dosage and attenuating side effects of CsA, and contribute to the treatment strategies of DES.

\section{Materials and methods}

\section{Experimental animals and ethics statement}

Thirty-two adult male New Zealand white rabbits with no signs of ocular inflammatory or gross abnormalities, weighing $2.0-2.5 \mathrm{~kg}$, were obtained from the animal facility of Fudan University, China. Rabbits were reared under standard laboratory conditions as follows: room temperature $22 \pm 2{ }^{\circ} \mathrm{C}$, relative humidity $60 \pm 10 \%$, and a 12-h light-dark cycle (0800 to 2000). All rabbits were quarantined and acclimatized a week before the experiment and free to food and water throughout the experiment. This study conformed to the standards and procedures for the proper care and use of animals, as described in the US Public Health Service Policy on Humane Care and Use of Laboratory Animals as well as the Association for Research in Vision and Ophthalmology Statement for the use of animals in ophthalmic and vision research. The protocol was approved by the committee on the ethics of animal experiments of Fudan Experimental Center. All operations and tests were performed under general anesthesia by an intramuscular injection of $40 \mathrm{mg} / \mathrm{kg}$ ketamine hydrochloride to keep the rabbits immobile and given an overdose of $80 \mathrm{mg} / \mathrm{kg}$ nembutal for euthanasia, with every effort to minimize suffering. No blinding research method was executed in the experiments.

\section{Induction of the DE models}

The left eyes of all the 32 rabbits were chosen for the induction of DE models and posterior experiments. Eye drops containing 0.3\% tobramycin (Alcon Inc., Fort Worth, TX, USA) were applied three times daily for 3 days preoperatively and all operations were performed under sterile conditions in the operating room for animals in Fudan Experimental Center after washing the conjunctival sac and ocular surface with povidone iodine. After instilling $0.5 \%$ proparacaine hydrochloride (Alcon Inc.) eye drops, an incision was made in the bulbar conjunctiva, the main lacrimal gland, Harderian gland and nictitating membrane were removed and each incision was sutured. Eye drops containing 0.3\% tobramycin and $0.1 \%$ dexamethasone (Alcon Inc.) four times daily, as well as an eye ointment of $0.3 \%$ tobramycin and $0.1 \%$ dexamethasone (Alcon Inc.) once a night for 7 days to prevent infection of eyes. Esculetin (St Louis, MO, USA) and/or 0.05\% CsA (Restasis; Allergan Inc., Irvine, CA, USA) eyedrops were administered 1 week after surgery and DES models were confirmed by a Schirmer I test (SIT) result of $\leq 10 \mathrm{~mm} / 5 \mathrm{~min}$ and/or tear-film break-up time (TBUT) $\leq 10 \mathrm{~s}$ without anesthetic eye drops and throughout the treatment period (8 weeks) for DES maintenance.

\section{Groups}

The 32 rabbits were randomly divided into four groups and the timing of the treatment eye drop instillation was 0800, 0000, 1400 and 1800: Group DES control $(n=8$; $100 \mu 1$ of phosphate buffer saline(PBS) topically applied quarter in die(QID) in left eyes); Group CsA ( $n=8 ; 100 \mu 1$ of $0.05 \%$ CsA topically applied QID in left eyes ); Group Esculetin $(n=8 ; 100 \mu$ l of $0.05 \%$ Esculetin, which was dissolved in PBS, topically applied QID in left eyes); Group CsA \& Esculetin (gourp C\&E, $n=8 ; 50 \mu 1$ of $0.05 \%$ CsA combined with $50 \mu$ l of $0.05 \%$ Esculetin topically applied QID in left eyes, with a 10 min interval between the instillations of different eyedrops); all the right eyes served as normal controls exposed to no solutions).

\section{Ocular tests}

The following ocular tests were performed pre-operation (week 0) and week 1, 2, 4, and 8 after induction of DES, at 1000 in the morning and repeated under the same conditions at 1600 in the afternoon, the average values of each test were calculated and recorded.

\section{Aqueous tear production}

The Schirmer's I test was carried out to determine the quantitative of aqueous tear production without anesthetic eye drops. The lower eyelid was slightly pulled down and the Schirmer's test strip (Tianjin Jingming New Technological Development Co., Ltd, China) was placed on the palpebral conjunctival vesica, located near the junction of the middle and outer third of the lower eyelid. After $5 \mathrm{~min}$, the wetting length of the strip was read and data were expressed in millimeters.

\section{Fluorescein on ocular surface}

After instilling one drop of $1 \%$ fluorescein (FL) solution (Tianjin Jingming New Technological Development Co., Ltd, China), eyes of all rabbits were examined under the 
slit-lamp microscope (Topcon SL-D7,Tokyo, Japan) with a cobalt blue filter at a magnification of 16 . The cornea was divided into four quadrants and the staining indensity in each quadrant was scored in a scale of $0-3$ and for a maximum score of 12 . Scoring was achieved using the following guidelines: no punctate staining on the cornea was assigned 0 point; punctate staining of 1-10 was assigned 1 point; punctate staining 11-30 was assigned 2 points; either punctate staining $>30$ or clumped staining was assigned 3 points. ${ }^{1}$

\section{Conjunctival impression cytology}

Conjunctival impression cytology (CIC) specimens were collected on week $0,1,2,4$, and 8 . Two $3.5 \times 3.5 \mathrm{~mm}$ circular disks of nitrocellulose filter paper (Pall Co., NY, USA) were placed separately on the nasal and temporal bulbar conjunctiva with the filter paper dull-side down. After the filter paper was pressed for $10 \mathrm{~s}$ with constant pressure, it was gently lifted and fixed with 95\% alcohol. Hematoxylin and periodic acid-Schiff (PAS) reagents were used to stain the specimens. After staining, the number of goblet cells was counted under a microscope (Olympus, Tokyo, Japan) with a 400 objective. Three different sections of each specimen were selected randomly for counting, and the average value was calculated as cells/high-power (HP) visual field with $\times 400)$.

\section{Tear fluid collection and determination of $I L-1 \alpha, I L-1 \beta$,} TNF- $\alpha$ by ELISA

To analyze inflammatory cytokine levels, tear samples were collected according to the method described previously. 7,8 Total of $50 \mu \mathrm{l}$ of PBS was instilled onto the ocular surface, while the animal was alert. The eyelid was manually blinked for several times and the fluids on the ocular surface were collected by sterile capillary tube. All of the tear samples were obtained from the lateral canthus, which parallel to the ocular surface, without stimulating reflex tearing, followed by immediate transfer to a $0.5 \mathrm{ml}$ Eppendorf tube and centrifugation at 1000 r.p.m. for $3 \mathrm{~min}$ at $4^{\circ} \mathrm{C}$. The supernatants then were stored at $-80^{\circ} \mathrm{C}$. The amount of tear IL- $1 \alpha$, IL- $1 \beta$, and TNF- $\alpha$ were measured using an enzyme-linked immunosorbent assay (ELISA) kit (eBioscience, San Diego, CA, USA) according to the manufacturer's instructions. The optical density of each well was determined at a wavelength of $450 \mathrm{~nm}$. Samples were considered positive when the signal was higher than the background signal (modified Krebs solution), and was within the range of the standard curve. The experiment was repeated thrice.

\section{Western blot analysis to detect $p E R K 1 / 2$ protein expression}

Western blotting was performed to analyze phosphorylated-ERK1/2 (p-ERK1/2) protein expression. All the rabbits were given an overdose of $80 \mathrm{mg} / \mathrm{kg}$ nembutal for euthanasia at week 8 . The lower bulbar conjunctivas were blunt desected and cut into small pieces for the determination. Specimens were stored at $-80^{\circ} \mathrm{C}$ and grindied into powder. Radio immunoprecipitation assay buffer $1 \mathrm{ml}$ and $10 \mu \mathrm{l}$ phenylmethyl sulfonylfluoride were added to each $100 \mathrm{mg}$ tissues on the $60 \mathrm{~Hz}$ tissue homogenate machine for $5 \mathrm{~min}$. The supernatants were obtained by centrifugation at $10,000 \mathrm{~g}$ for $15 \mathrm{~min}$ at $4^{\circ} \mathrm{C}$. The protein concentrations in the lysate were quantified by the BCA protocols. The proteins were resolved by 10\% SDSpolyacrylamide gel electrophoresis (SDS-PAGE) and transferred onto a nitrocellulose membrane. The membranes were blocked with 5\% nonfat milk in TBST (pH 7.6) for $1 \mathrm{hr}$, incubated overnight at $4^{\circ} \mathrm{C}$ with anti-pERK1/2 antibody (mouse anti-rabbit IgG, NO: 4370S; Cell Signaling Technology Inc., Boston, MA, USA), diluted $1: 1000$, washed extensively and then incubated with secondary antibody (1:5000, goat anti-mouse IgG, No: 7076; Cell Signaling Technology Inc.) for $2 \mathrm{~h}$. The bands were visualized by chemiluminescence and observed at an optimal timepoint. Protein bands were quantified by densitometric analysis using GENE TOOL (UVP). The Western blot was triplicated.

\section{Analysis}

Statistical analysis was performed using SPSS17.0 software (SPSS Inc., Chicago, IL, USA). All data were presented as means \pm SD. Values were compared among the groups and time points using analysis of one-way ANOVA, and $P<0.05$ was considered statistically significant.

\section{Results}

\section{Schirmer's I test}

No significant difference in aqueous tear production was identified in each group at week $0(P>0.05)$. After induction of DES, the wetting length of Schirmer's test strip at week $1,2,4$, and $8(8 \pm 3 \mathrm{~mm}, 9 \pm 2 \mathrm{~mm}, 9 \pm 2 \mathrm{~mm}$, $8 \pm 2 \mathrm{~mm})$ was shorter than week $0(P<0.01)$. Schirmer's I test (SIT) values of group CsA $(11 \pm 2 \mathrm{~mm})$, group Esculetin $(9 \pm 3 \mathrm{~mm})$, and group C\&E $(12 \pm 2 \mathrm{~mm})$ were obviously higher than group DES control since week 1 $(P<0.05)$. Meanwhile, SIT of group C\&E was increased the most at any timespot when compared with groups CsA and group Esculetin $(P<0.05)$, and the highest SIT 
values of the three therapy groups $(12 \pm 1 \mathrm{~mm}, 10 \pm 2 \mathrm{~mm}$, $14 \pm 1 \mathrm{~mm}$ ) were observed at week 4 (Figure 1 ).

\section{Cornea fluorescein scores}

Photographs were obtained at week 0 and 8 in each group (Figures 2a-j). There was no significant difference in minimal corneal punctuate staining in each group at baseline (Figures $2 \mathrm{a}-\mathrm{e}, P>0.05)$. In the normal control group, no apparent changes in scores were observed throughout the study, while FL scores were significantly increased after operation in other four groups (Figure 2k, $P<0.01)$. FL scores in group DES $(9.33 \pm 0.33)$ were higher than group CsA (7.92 \pm 0.56$)$, group Esculetin $(8.62 \pm 0.32)$ and group C\&E $(6.71 \pm 0.26)$ since week 1 (Figure $2 \mathrm{k}$, $P<0.05)$. Clumped FL staining was still shown on cornea in group DES (Figure 2g) and punctuate staining in each quadrant of cornea in group Esculetin (Figure 2i). Scores in three therapy groups decreased obviously after instilling different drugs and the lowest results were found in group C\&E at week 1, 2, 4, and 8 (Figure 2k, $P<0.05)$. FL scores in group CsA were lower than group Esculetin from week 1 to week 8 (Figure $2 k, P<0.05$ ).

\section{Goblet cell density}

Assessment of the CIC focused on goblet cell density (Table 1). There was no difference in goblet cell number in

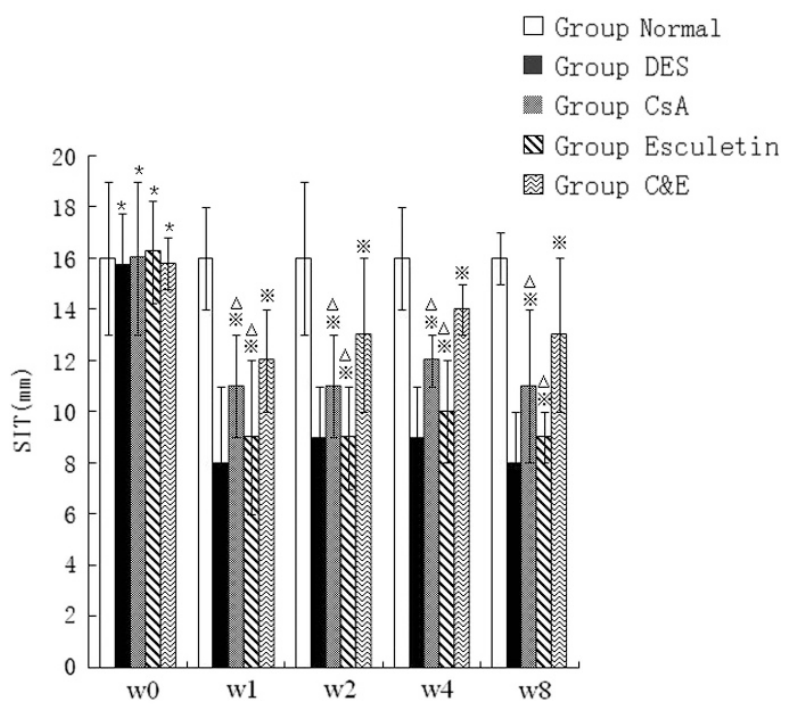

Figure 1 Comparison of the wetting length of Schirmer I test at different time points. Wetting length of Schirmer's test strip was shorter than week 0 after induction of DES and increasing of SIT was observed after therapy from week 1 to week 8 in group CsA, group Esculetin and group $C \& E$. $\left({ }^{*} P<0.05\right.$ compared with group Normal control at week $0,{ }^{*} P<0.05$ compared with group DES at week $1-8$ respectively, $\triangle P<0.05$ compared with group $C \& E$ at week $1-8$, respectively). each group preoperative at baseline $(P<0.05)$. Goblet cell density in group DES decreased to $72 \pm 21$ cells $/ \mathrm{HP}$ at week 1 and $50 \pm 14$ cells/HP at week 8 . Meanwhile, both group CsA and group Esculetin alleviate decreasing percentage of goblet cell density, respectively $54.83 \pm 6.72 \%$ and $52.75 \pm 8.14 \%$ at week 8 , which showed no significant difference $(P>0.05)$ between the two groups. However, obvious increase of goblet cell density were observed at any time spot in group C\&E when compared with group CsA and group Esculetin $(P<0.05)$.

\section{Concentrations of $I L-1 \alpha, I L-1 \beta$, and TNF- $\alpha$}

The concentrations of IL- $1 \alpha, \mathrm{IL}-1 \beta$, and TNF- $\alpha$ were detected by ELISA to estimate the chronic inflammation on ocular surface (Table 2). No significant difference was found of IL- $1 \alpha$, IL- $1 \beta$, and TNF- $\alpha$ concentrations in each group at week $0(P>0.05)$. As shown in the tables, DE resulted in higher levels of IL- $1 \alpha$, IL- $1 \beta$, and TNF- $\alpha$. IL- $1 \alpha$ and IL-1 $\beta$ concentrations increased gradually during the study $(P<0.05)$, TNF- $\alpha$ increased from week 1 to week 4 , and then decreased at week $8(P<0.05)$. Upregulations of IL- $1 \alpha$, IL-1 $\beta$, and TNF- $\alpha$ were inhibited in group CsA, group Esculetin and group $C \& E(P<0.05)$. The maximum inhibiting effect was noted in group $C \& E(P<0.05)$.

\section{Expression of pERK1/2 protein}

Immunoblotting analysis was performed to determine the levels of $\mathrm{p}$-ERK1/2 protein in each group before and after therapy. DE activated the ERK1/2 signal pathway and the bands in group DES were much more stronger(gray value was 2.35 fold than GAPDH) than other groups (Figure 3a). Upregulations of p-ERK1/2 in group CsA and group Esculetin were observed and the bands in group CsA were stronger than that in group Esculetin, and gray values were 1.87 fold and 1.44 fold than GAPDH respectively and reduced to 1.12 fold in group $C \& E$ (Figure $3 b, P<0.05$ ). The difference were obviously revealed among the three therapy groups and the most decreasing of $\mathrm{p}$-ERK1/2 expression was observed in group $\mathrm{C} \& \mathrm{E}(P<0.05)$.

\section{Discussion}

Human tear film coats the anterior ocular surface and is composed of three distinct layers: an inner mucin coating, a middle aqueous component, a lipid overlay and the aqueous component is derived from the lacrimal gland. ${ }^{2}$ The lacrimal gland fluid contributes $58-79 \%$ of the total volume of tears in rabbits. ${ }^{9}$ The secretory cells of the lacrimal gland produce a highly complex product of water, ions, and proteins, ${ }^{10}$ which are the main components of the aqueous layer. In this study, accessory 

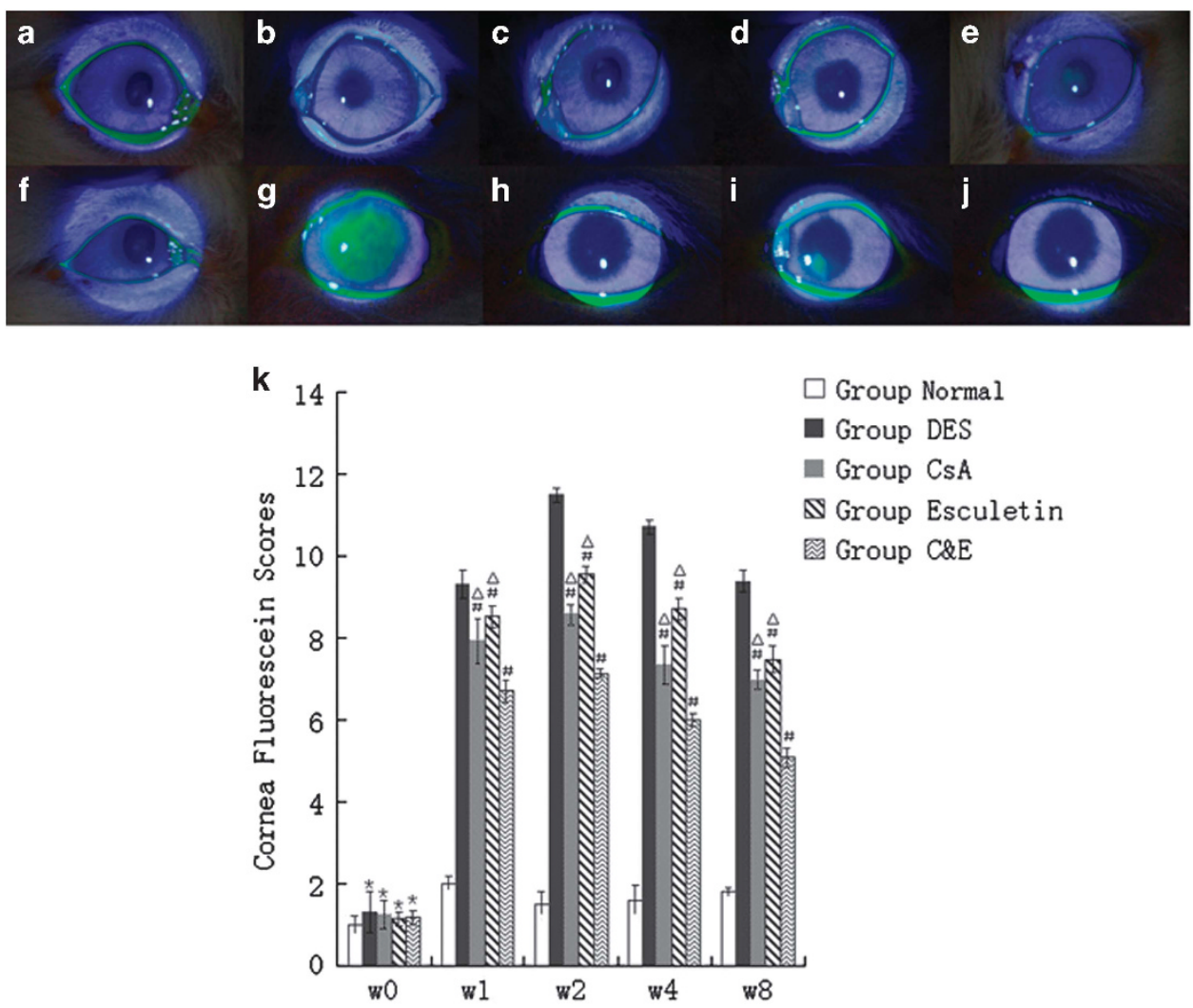

Figure 2 Cornea fluorescein staining observed under the slit-lamp microscope with cobalt blue filter at a magnification of 16. (a-e) were figures observed at week 0 (a) group normal control; (b) group DES; (c) group CsA; (d) group Esculetin; (e) group (c and e). No significant difference on the ocular surface was shown among groups. $(\mathrm{f}-\mathrm{j})$ were figures observed at week 8 (f) group normal control; ( $\mathrm{g}$ ) group DES; (h) group CsA; (i) group Esculetin; (j) group (c and e). Clumped staining was observed in (g), punctate staining diminished in (i) and nearly vanished in (h and j). Cornea fluorescein scores of groups at week 0 to week 8 were shown in (k). There was no significant difference in each group preoperatively and the scores increased after establishing of DE models and were persistent. The decreasing of FL scores were observed after therapy with CsA or/and Esculetin eyedrops instilling. $\left({ }^{*} P<0.05\right.$ compared with group Normal control at week $0,{ }^{\#} P<0.05$ compared with group DES at week $1-8$ respectively, $\triangle P<0.05$ compared with group C\&E at week $1-8$, respectively).

Table 1 Goblet cell density calculated with microscope at magnification of 400

\begin{tabular}{lccccc}
\hline & Week 0 & Week 1 & Week 2 & Week 4 & Week 8 \\
\hline GroupNormal & $140 \pm 23$ & $145 \pm 19$ & $138 \pm 17$ & $142 \pm 11$ & $145 \pm 13$ \\
Group DES & $141 \pm 25$ & $72 \pm 21^{*}$ & $55 \pm 16^{*}$ & $59 \pm 15^{*}$ & $50 \pm 14^{*}$ \\
Group CsA & $138 \pm 18^{*}$ & $108 \pm 20^{\#} \Delta$ & $86 \pm 10^{\#} \triangle$ & $81 \pm 10^{\# \Delta}$ & $77 \pm 12^{\# \triangle}$ \\
Group Esculetin & $143 \pm 19^{*}$ & $110 \pm 17^{\# *}$ & $88 \pm 13^{\#} \triangle *$ & $83 \pm 13^{\# \Delta *}$ & $74 \pm 12^{\# \Delta *}$ \\
Group C\&E & $137 \pm 17^{*}$ & $126 \pm 25^{\#}$ & $109 \pm 15^{\#}$ & $101 \pm 14^{\#}$ & $92 \pm 12^{\#}$ \\
\hline
\end{tabular}

${ }^{*} P<0.05$ compared with group Normal control at week 0-8.

${ }^{\#} P<0.05$ compared with group DES at week $1-8$.

$\triangle P<0.05$ compared with group C\&E.

${ }^{*} P<0.05$ compared with group CsA at week $1-8$.

lacrimal glands in the superior palpebral conjunctiva of rabbits are reserved, which acinar systems have cellular features that resemble of the main lacrimal glands. ${ }^{11}$ Sjögren's syndrome (SS) is a chronic and progressive systemic autoimmune disease that primarily involves immune-mediated damage to the lacrimal and salivary glands. ${ }^{1,12}$ This pathology translates into dry eyes (kerato conjunctivitis sicca) and dry mouth. SS-induced dry eye is one of the primary type of DES. We established the DE models, which were extremely imitating the ocular surface condition of SS-induced dry eye. Objective ocular tests can help diagnosing or monitoring of dry eye. ${ }^{4}$ In group DES control, obvious alternations of the SIT and FL scores and symptoms after establishing the model 
Table 2 IL- $1 \alpha, \mathrm{IL}-1 \beta$ and TNF- $\alpha$ expressions detected by ELISA

\begin{tabular}{|c|c|c|c|c|c|}
\hline & Week 0 & Week 1 & Week 2 & Week 4 & Week 8 \\
\hline \multicolumn{6}{|l|}{$I L-1 \alpha(p g / m l)$} \\
\hline GroupNormal & $5.13 \pm 0.54$ & $5.01 \pm 0.34$ & $4.98 \pm 0.32$ & $5.19 \pm 0.59$ & $5.07 \pm 0.47$ \\
\hline Group DES & $5.08 \pm 0.62^{*}$ & $7.87 \pm 0.96^{*}$ & $9.57 \pm 1.37^{*}$ & $11.56 \pm 2.33^{*}$ & $13.55 \pm 2.89^{*}$ \\
\hline Group CsA & $5.21 \pm 0.34^{*}$ & $5.98 \pm 0.86^{\# \triangle}$ & $6.73 \pm 0.99^{\# \triangle}$ & $7.37 \pm 1.01^{\# \triangle}$ & $8.56 \pm 1.67^{\# \triangle}$ \\
\hline Group Esculetin & $5.28 \pm 0.69^{*}$ & $6.92 \pm 1.01^{\# \triangle}$ & $7.85 \pm 1.17^{\# \triangle}$ & $9.33 \pm 1.58^{\# \triangle}$ & $8.92 \pm 1.04^{\# \triangle}$ \\
\hline Group C\&E & $5.15 \pm 0.57^{*}$ & $5.45 \pm 0.57^{\#}$ & $5.94 \pm 0.60^{\#}$ & $6.29 \pm 0.77^{\#}$ & $6.81 \pm 0.69^{\#}$ \\
\hline \multicolumn{6}{|l|}{$I L-1 \beta(p g / m l)$} \\
\hline Group Normal & $4.17 \pm 0.32$ & $4.05 \pm 0.19$ & $4.21 \pm 0.41$ & $4.37 \pm 0.39$ & $4.19 \pm 0.30$ \\
\hline Group DES & $4.26 \pm 0.24^{*}$ & $6.28 \pm 0.51^{*}$ & $8.53 \pm 0.87^{*}$ & $10.11 \pm 1.07^{*}$ & $12.53 \pm 2.19^{*}$ \\
\hline Group CsA & $4.01 \pm 0.13^{*}$ & $5.46 \pm 0.49^{\# \triangle}$ & $6.35 \pm 0.67^{\# \triangle}$ & $7.02 \pm 0.71^{\# \triangle}$ & $7.95 \pm 0.91^{\# \triangle}$ \\
\hline Group Esculetin & $4.23 \pm 0.38^{*}$ & $5.98 \pm 0.54^{\# \triangle}$ & $6.71 \pm 0.59^{\# \triangle}$ & $8.82 \pm 0.76^{\# \triangle}$ & $9.37 \pm 1.25^{\# \triangle}$ \\
\hline Group C\&E & $4.14 \pm 0.37^{*}$ & $5.15 \pm 0.56^{\#}$ & $5.88 \pm 0.62^{\#}$ & $6.51 \pm 0.67^{\#}$ & $6.72 \pm 0.58^{\#}$ \\
\hline \multicolumn{6}{|l|}{$T N F-\alpha(p g / m l)$} \\
\hline Group Normal & $13.81 \pm 2.01$ & $14.11 \pm 2.23$ & $13.75 \pm 1.94$ & $13.92 \pm 2.00$ & $14.25 \pm 2.31$ \\
\hline Group DES & $13.42 \pm 1.93^{*}$ & $19.87 \pm 3.16^{*}$ & $25.32 \pm 4.01^{*}$ & $37.59 \pm 4.31^{*}$ & $35.92 \pm 3.57^{*}$ \\
\hline Group CsA & $14.02 \pm 1.57^{*}$ & $16.17 \pm 1.89^{\# \triangle}$ & $18.97 \pm 2.03^{\# \triangle}$ & $19.54 \pm 2.12^{\# \triangle}$ & $19.63 \pm 1.78^{\# \triangle}$ \\
\hline Group Esculetin & $13.94 \pm 1.26^{*}$ & $19.13 \pm 2.91^{\# \triangle}$ & $21.67 \pm 3.11^{\# \triangle}$ & $20.09 \pm 2.34^{\# \triangle}$ & $20.98 \pm 2.02^{\# \triangle}$ \\
\hline Group C\&E & $13.28 \pm 0.96^{*}$ & $14.29 \pm 1.54^{\#}$ & $16.12 \pm 1.79^{\#}$ & $16.78 \pm 1.59^{\#}$ & $16.85 \pm 1.46^{\#}$ \\
\hline
\end{tabular}

${ }^{*} P<0.05$ compared with group Normal control.

${ }^{\#} P<0.05$ compared with group DES.

$\triangle P<0.05$ compared with group C\&E.

a

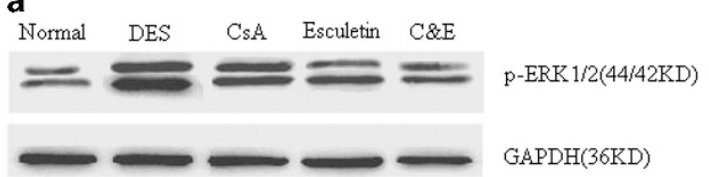

b

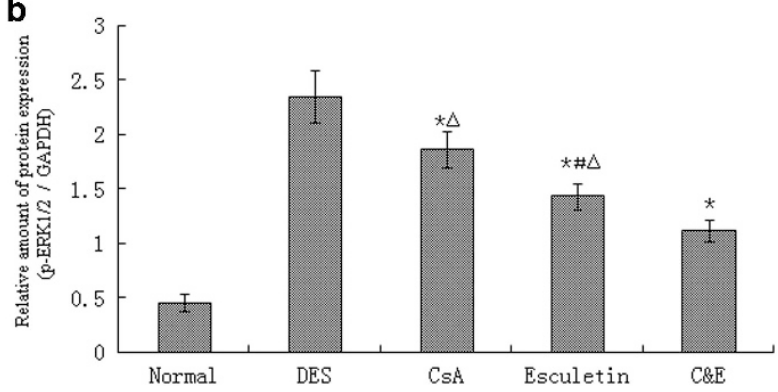

Figure 3 Western blot analysis of p-ERK1/2 protein in group Normal, DES, CsA, Esculetin and C\&E. (a) Representative Western blots. (b) Quantification of p-ERK1/2 in each group using densitometric analysis of the bands shown in (a). Expression levels values normalized to GAPDH levels. $\left({ }^{*} P<0.05\right.$ compared with group DES, ${ }^{\#} P<0.05$ compared with group CsA, ${ }^{\#} P<0.05$ compared with group $\left.C \& E\right)$.

continued during the whole experimental period (Figure 1). SIT valve was $15.75 \pm 2 \mathrm{~mm}$ before surgery and reduced to $8 \pm 3 \mathrm{~mm}$ in week 1 . Tear secretion and tear film may be related to either compensation of neurally regulated conjunctival goblet cell mucin secretion ${ }^{13}$ or secretion of the accessory lacrimal gland on the conjunctiva.
Cyclosporine A (CsA) is a neutral, hydrophobic, cyclic peptide of amino acids which was isolated from several species of fungi ${ }^{14}$ and used for corneal graft rejection, autoimmune uveitis, and dry eye syndrome ${ }^{15}$ through suppressing cell-mediated immune responses. ${ }^{16,17}$ The mechanism based on its effects on subconjunctival and lacrimal gland inflammation, resulting in an increase in tear production, and conjunctival goblet cell density. 3,18 Ophthalmic CsA has been available for a few years and beneficial in DES, which recommended concentration is $0.05 \%$, could slow down or prevent the progression of DES. ${ }^{19}$ It is important to consider both the benefits and risks of dry eye therapy and select the treatment appropriately. Esculetin is a derivative of coumarin that is present in Chinese herbal drug 'Qinpi', used as folk medicines, such as Fraxinus rhynchophylla, Rehmanniae glutinosa and Artemisia capillaries. ${ }^{20-23}$ Previously, the inhibitory effect of Esculetin on cell proliferation in human cell lines had been reported. ${ }^{24,25}$ Esculetin reduced CCl4-induced hepatic apoptosis in rats probably against hepatocellular apoptosis with its free-radical scavenging ability. ${ }^{26}$ It was also suggested to be a potent cancer therapeutic and preventive agent. Lee et al identified Esculetin as a potent inhibitor of Wnt signaling, decreased viability, inhibited anchorage-independent growth of colon cancer cells and tumor growth in a xenograft mouse model. ${ }^{27}$ Esculetin was capable of inhibiting growth of hepatocellular carcinoma both in vivo and in vitro, it also resulted in Bax/Bcl-2 ratio, activation of the caspase cascade and loss of MMP. ${ }^{28}$ Topical Esculetin on ocular 
surface had never been reported ever before. We administrated $0.05 \%$ Esculetin solution which concentration was identical to the CsA to the DE rabbitsand found that Esculetin have effectively increased SIT values $(9 \pm 3 \mathrm{~mm})$, goblet cell densities $(110 \pm 17$ cells / $\mathrm{HP})$ and decreased FL scrores $(9.12 \pm 0.32)$ since week 1. Effects of Esculetin continuously maintained till week 8, while the SIT values were $9 \pm 1 \mathrm{~mm}$, goblet cell densities were $74 \pm 12$ cells $/ \mathrm{Hp}$ and FL scores were $7.48 \pm 0.33$.

These results were superior to that in group DES control, even though the extent was moderate than CsA or Esculetin combining with CsA (Figures 1, 2 and Table 1). We can also estimate that Esculetin improve DE sympotoms through stimulating aqueous secretion of accessory lacrimal gland or neurally regulated conjunctival goblet cell mucin secretion.

There are many cellular and molecular process involved in the pathogenesis of dry eye, including increased inflammatory cytokines, tear film osmolarity, metalloproteinases, chemokines and their receptors, inflammatory cascades, and activation of immune cells. ${ }^{29}$ The major pro-inflammatory cytokines found to be important in pSS are interferons, IL-12, IL-18, TNF $\alpha$, IL-1 $\beta$, IL-6, IL-17, IL-23 and B-cell activating factor (BAFF). ${ }^{30}$ There was profound increment of IL-4 and IL-5, which were produced by T helper2 (Th2) cells, in the salivary proteome of patients with pSS. ${ }^{31}$ Cai et al ${ }^{32}$ found that Esculetin inhibit IL-6 expression and it could also inhibit NO, TNF- $\alpha$, IL-1 $\beta$ effectively when combinated with curcumin and hesperetin. IL- 6 and IL- 8 production were prevented with Esculetin in LPS-induced rat intestinal microvascular endothelial cells. ${ }^{33} \mathrm{We}$ investigated the IL- $1 \alpha$, IL-1 $\beta$, TNF $\alpha$ concentrations of tear samples in ELISA essay and gradual increasing of all the three kinds of cytokines were shown from week 1 to week 8 in group DES control. IL- $1 \alpha$, IL- $1 \beta$, TNF $\alpha$ concentrations were gradually increased in three therapy groups at relatively low amplitude (Table 2). Therefore,effects of topical Esculetin and/or CsA were detected obviously in this essay and the most expecting results appeared in group C\&E. This was not only proving Esculetin inhibit expressions of some interleiukins, but also reveal that Esculetin exhance the anti-inflammatory function of CsA.

ERK cascade plays a crucial role in multiple cellular processes such as cell proliferation, differentiation, adhesion, migration, survival and apoptosis. ${ }^{34,35}$ ERK1/2 pathway participated in the pro-inflammatory responses mounted by pSS salivary gland epithelial cells. ${ }^{36}$ Experimental induced dry eye in mice stimulated expression and production of IL- $1 \beta$, TNF- $\alpha$, MMP-9, and avtivated ERK1/2 signaling pathway on the ocular surface, ${ }^{37}$ some growth factor besides EGF stimulated conjunctival goblet cell proliferation by ERK1/2 pathway. ${ }^{38}$ Dryness also increased expressions of IL- $1 \alpha$,
IL-1 $\beta$ and TNF- $\alpha$ mRNA and stimulated phosphorylation of JNK1/2, ERK1/2 and p38 MAPKs in the corneal epithelium ${ }^{39}$ and even activation of several signaling pathway, including ERK in RPE-choroid AMD phenotypes and MAPK inhibitors could provide a novel therapeutic strategy for prevention or treatment of AMD. ${ }^{40,41}$ In normal control group, gray value of p-ERK1/2 was 0.46 fold than GAPDH, while it was 2.31 fold in group DES. This once again verified the correlation between chronic ocular surface inflammation and ERK1/2 signal pathway. When refer to the therapy groups, gray values were respectively 1.87 fold in group CsA, 1.44 fold in group Esculetin and 1.12 fold in group C\&E (Figure 3). Results indicated suppression of ERK1/2 pathway after different therapy, consistented with observations in the former parts of the study and confirmed the effect of Esculetin combining with CsA. We found that $\mathrm{p}$-ERK1/2 expression in group Esculetin was nearly $30 \%$ lower than group CsA. It was maybe because of ERK1/2 pathway play a more important role in DES treating with Esculetin, rather than with CsA.

In conclusion, we topically applicated Esculetin firstly to search for a new potential drug for the patient suffering from DES. The results of ocular tests, cytokine levels and specific phosphorylated protein of ERK1/2 signal pathway proved that Esculetin has resemble therapeutic effect on curing DES when compared with CsA. Esculetin was capable of enhancing the effect of CsA and meanwhile it was more available and in lower price in eastern countries. We only observed the ERK1/2 pathway in this article and whether there exist other pathways crosstalking with it remains uncertain. All these encourage us to approaching for the concrete mechanism and most effective concentration of topical Esculetin in anti-inflammatory of DES.

\section{Summary}

What was known before

- High prevelance of dry eye syndrome in aged people was noticed and its clinical manifestation is chronic inflammation on the ocular surface. CsA can improve the symptoms and signs of dry eye, however, it has side effects.

What this study adds

- Topical Esculetin improves DE symptoms, downregulats the inflammatory cytokine expressions, suppresses the ERK1/2 signal pathway and enhances the therapeutic effect of CsA.

\section{Conflict of interest}

The authors declare no conflict of interest. 


\section{Acknowledgements}

We gratefully acknowledge Fudan Animal Experimental Center for supporting sterile experimental operation room, technical assistance in animal rearing and sample testing, Xiaofei Song (Department of Urology, Minhang Affiliated Hospital of Fudan University) for data statistical analysis, Zhaoyang Wang (Department of Ophthalmology, Shanghai No. 9 Hosptial) for native English instructions. This work was supported by the Natural Science Foundation of China (No. 81574028), Shanghai Health Bureau of Traditional Chinese Medicine Scientific Fund (No. 2012J005A) and Longhua Medical Scholar (LYTD-50).

\section{References}

1 The definition and classification of dry eye disease: report of the Definition and Classification Subcommittee of the International Dry Eye WorkShop. Ocul Surf 2007; 5(2): 75-92.

2 Conrady CD, Joos ZP, Patel BC. Review: the lacrimal gland and its role in dry eye. J Ophthalmol 2016; 2016: 7542929.

3 Sall K, Stevenson OD, Mundorf TK, Reis BL. Two multicenter, randomized studies of the efficacy and safety of cyclosporine ophthalmic emulsion in moderate to severe dry eye disease. CsA Phase 3 Study Group. Ophthalmology 2000; 107(4): 631-639.

4 Kim AD, Madduma Hewage SR, Piao MJ, Kang KA, Cho SJ, Hyun JW. Esculetin induces apoptosis in human colon cancer cells by inducing endoplasmic reticulum stress. Cell Biochem Funct 2015; 33(7): 487-494.

5 Yum S, Jeong S, Lee S, Kim W, Nam J, Jung Y. HIF-prolyl hydroxylase is a potential molecular target for esculetinmediated anti-colitic effects. Fitoterapia 2015; 103(6): 55-62.

6 Park SL, Won SY, Song JH, Lee SY, Kim WJ, Moon SK. Esculetin Inhibits VEGF-Induced angiogenesis both in vitro and in vivo. Am J Chin Med 2016; 44(1): 61-76.

7 Lee SY, Han SJ, Nam SM, Yoon SC, Ahn JM, Kim TI et al. Analysis of tear cytokines and clinical correlations in Sjögren syndrome dry eye patients and non-Sjögren syndrome dry eye patients. Am J Ophthalmol 2013; 156(2): 247-253.e1.

8 Zhou L, Wei R, Zhao P, Koh SK, Beuerman RW, Ding C. Proteomic analysis revealed the altered tear protein profile in a rabbit model of Sjögren's syndrome-associated dry eye. Proteomics 2013; 13(16): 2469-2481.

9 Thörig L, van Agtmaal EJ, Glasius E, Tan KL, van Haeringen NJ. Comparison of tears and lacrimal gland fluid in the rabbit and guinea pig. Curr Eye Res 1985; 4(8): 913-920.

10 Walcott B. The lacrimal gland and its veil of tears. News Physiol Sci 1998; 13: 97-103.

11 Bergmanson JP, Doughty MJ, Blocker Y. The acinar and ductal organisation of the tarsal accessory lacrimal gland of Wolfring in rabbit eyelid. Exp Eye Res 1999; 68(4): 411-421.

12 Tincani A, Andreoli L, Cavazzana I, Doria A, Favero M, Franceschini F et al. Novel aspects of Sjögren's syndrome in 2012. BMC Med 2013; 4(11): 93.

13 Dartt DA. Regulation of mucin and fluid secretion by conjunctival epithelial cells. Prog Retin Eye Res 2002; 21(6): $555-576$.
14 Petcher TJ, Weber H, Rueegger A. Crystal and molecular structure of an iodo derivative of the cyclic undecapeptide cyclosporin A. Helv Chim Acta 1976; 59(5): 1480-1489.

15 Nussenblatt RB, Palestine AG, Chan CC, Stevens Jr G, Mellow SD, Green SB. Randomized, double-masked study of cyclosporine compared to prednisolone in the treatment of endogenous uveitis. Am J Ophthalmol 1991; 112(2): 138-146.

16 Noble S, Markham A. Cyclosporin. A review of the pharmacokinetic properties, clinical efficacy and tolerability of a microemulsion-based formulation (Neoral). Drugs 1995; 50(5): 924-941.

17 Donnenfeld E, Pflugfelder SC. Topical ophthalmic cyclosporine: pharmacology and clinical uses. Surv Ophthalmol 2009; 54(3): 321-338.

18 Wilson SE, Perry HD. Long-term resolution of chronic dry eye symptoms and signs after topical cyclosporine treatment. Ophthalmology 2007; 114(1): 76-79.

19 Yavuz B, Bozdag Pehlivan S, Unlu N. An overview on dry eye treatment: approaches for cyclosporine a delivery. ScientificWorldJournal 2012; 2012: 194848.

20 Hong GE, Park HS, Kim JA, Nagappan A, Zhang J, Kang SR et al. Anti-oxidant and anti-inflammatory effects of Fraxinus rhynchophylla on lipopolysaccharide (LPS)-induced murine Raw 264.7 cells. J Biomed Res 2012; 13(4): 331-338.

21 Wu ZB, Liu Y, Tian SS, Wen C. Chemical constituents of the stem bark of Fraxinus rhynchophylla. Chem Nat Compd 2014; 49(6): 1162-1163.

22 Si CL, Liu Z, Su YF, Kim JK, Bae YS. Coumarins and secoiridoid glucosides from bark of Fraxinus rhynchophylla Hance. Holzforschung 2008; 62(5): 553-555.

23 Prabakaran D, Ashokkumar N. Protective effect of esculetin on hyperglycemia-mediated oxidative damage in the hepatic and renal tissues of experimental diabetic rats. Biochimie 2013; 95(2): 366-373.

$24 \mathrm{Ng} \mathrm{TB}$, Liu F, Wang ZT. Antioxidative activity of natural products from plants. Life Sci 2000; 66(8): 709-723.

25 Adfa M, Yoshimura T, Komura K, Koketsu M. Antitermite activities of coumarin derivatives and scopoletin from Protium javanicum Burm. J Chem Ecol 2010; 36(7): 720-726.

26 Tien YC, Liao JC, Chiu CS, Huang TH, Huang CY, Chang WT et al. Esculetin ameliorates carbon tetrachloridemediated hepatic apoptosis in rats. Int J Mol Sci 2011; 12(6): 4053-4067.

27 Lee SY, Lim TG, Chen H, Jung SK, Lee HJ, Lee MH et al. Esculetin suppresses proliferation of human colon cancer cells by directly targeting $\beta$-catenin. Cancer Prev Res (Phila) 2013; 6(12): 1356-1364.

28 Wang J, Lu ML, Dai HL, Zhang SP, Wang HX, Wei N. Esculetin, a coumarin derivative, exerts in vitro and in vivo anti proliferative activity against hepatocellular carcinoma by initiating a mitochondrial-dependent apoptosis pathway. Braz J Med Biol Res 2015; 48(3): 245-253.

29 Coursey TG, de Paiva CS. Managing Sjögren's syndrome and non-Sjögren's syndrome dry eye with anti-inflammatory therapy. Clin Ophthalmol 2014; 8: 1447-1458.

30 Roescher N, Tak PP, IIIei GG. Cytokines in Sjögren's syndrome. Oral Dis 2009; 15(8): 519-526.

31 Delaleu N, Mydel P, Kwee I, Brun JG, Jonsson MV, Jonsson R. High fidelity between saliva proteomics and the biologic state of salivary glands defines biomarker signatures for primary Sjögren's syndrome. Arthritis Rheumatol 2015; 67(4): 1084-1095.

32 Cai C, Chen Y, Zhong S, Zhang Y, Jiang J, Xu H et al. Synergistic effect of compounds from a Chinese herb: 
compatibility and dose optimization of compounds from N-Butanol extract of lpomoea stolonifera. Sci Rep 2016; 6: 27014.

$33 \mathrm{Hu}$ Y, Chen X, Duan H, Hu Y, Mu Y. Chinese herbal medicinal ingredients inhibit secretion of IL-6, IL-8, E-selectin and TXB2 in LPS-induced rat intestinal microvascular endothelial cells. Immunopharmacol Immunotoxicol 2009; 31(4): 550-555.

34 Yoon S, Seger R. The extracellular signal-regulated kinase: multiple substrates regulate diverse cellular functions. Growth Factors 2006; 24(1): 21-44.

35 Busca R, Pouyssegur J, Lenormand P. ERK1 and ERK2 map kinases: specific roles or functional redundancy. Front Cell Dev Biol 2016; 4: 53.

36 Sisto M, Lisi S, D'Amore M, Lofrumento DD. The metalloproteinase ADAM17 and the epidermal growth factor receptor (EGFR) signaling drive the inflammatory epithelial response in Sjögren's syndrome. Clin Exp Med 2015; 15(2): 215-225.

37 Luo L, Li DQ, Doshi A, Farley W, Corrales RM, Pflugfelder SC. Experimental dry eye stimulates production of inflammatory cytokines and MMP-9 and activates MAPK signaling pathways on the ocular surface. Invest Ophthalmol Vis Sci 2004; 45(12): 4293-4301.

38 Li D, Shatos MA, Hodges RR, Dartt DA. Role of PKCoactivation of Src, PI-3K/AKT, and ERK in EGF-stimulated proliferation of rat and human conjunctival goblet cells. Invest Ophthalmol Vis Sci 2013; 54(8): 5661-5674.

39 De Paiva CS, Corrales RM, Villarreal AL, Farley WJ, Li DQ, Stern ME et al. Corticosteroid and doxycycline suppress MMP-9 and inflammatory cytokine expression, MAPK activation in the corneal epithelium in experimental dry eye. Exp Eye Res 2006; 83(3): 526-535.

40 Makarev E, Cantor C, Zhavoronkov A, Buzdin A, Aliper A, Csoka AB. Pathway activation profiling reveals new insights into age-related macular degeneration and provides avenues for therapeutic interventions. Aging (Albany NY) 2014; 6(12): 1064-1075.

41 SanGiovanni JP, Lee PH. AMD-associated genes encoding stress-activated MAPK pathway constituents are identified by interval-based enrichment analysis. PLoS One 2013; 8(8): e71239. 